\title{
Home-Made Vitrification and Thawing Media Did Not Differ Than a Commercially Available Brand on Blastocyst Survival, Expansion and Implantation
}

Original

Ahmed Samy Saad ${ }^{1,2}$, Sahar Afify Eissa ${ }^{2}$,Walid Abdel-Latif Abdel-Halim ${ }^{2,3}$

${ }^{1}$ Department of Obstetrics and Gynecology, ${ }^{3}$ Department of Clinical and Chemical pathology, Faculty of Medicine, Banha University, ${ }^{2}$ Hawa Fertility Center, Banha, Egypt

\begin{abstract}
Aim: This study was conducted to compare the cryo-survival and implantation rates of day $5 / 6$ human blastocysts after using a home-made media versus a commercial freezing and thawing kit.

Materials and Methods: This is a prospective comparative study. Group 1 blastocysts were vitrified with home-made media $(\mathrm{HM})(\mathrm{n}=48)$ according to shady grove protocol and thawed with gradual thawing technique using HM according to Kuwayama protocol. Group $2(\mathrm{n}=30)$ blastocysts were vitrified and thawed with commercial media $(\mathrm{CM})$. Blastocysts were subsequently cultured for 6-8 h, assessed for survival and expansion, then embryo transfer. The percentages of Positive hCG, clinical pregnancy, implantation, multiple pregnancy and abortion were recorded.

Results: Survival (following thawing and after 6-8 h culture), re-expansion, and implantation rate as well as the pregnancy rate were calculated for both media. Pregnancy rate for $\mathrm{CM}$ was $70 \%(\mathrm{n}=21 / 30)$ and $70.83 \%(\mathrm{n}=34 / 48 ; \mathrm{P}=0.8)$ for HM. Implantation rate for CM was $60 \%(n=18 / 30)$ and $60.42 \%(n=29 / 48 ; P=0.9)$ for HM. There were no significant differences between the two groups. Both solutions provided high survival rate and of total 47 cases who recorded implantation, 45 went home with Healthy babies.

Conclusion: The two vitrification protocols, commercial kit and home-made solution, did not differ in blastocysts survival, re-expansion and implantation rates. So both methods could be used with equal success. HM it is providing an equal safety, and efficiency with lower cost in compared to CM.
\end{abstract}

Key Words: Blastocyst survival, home-made vitrification, thawing media, vitrification

Received: 02 July 2020, Accepted: 14 December 2020

Corresponding Author: Ahmed Samy Saad, Department of Obstetrics and Gynecology, Faculty of Medicine, Banha University, Qalyubia, Egypt, Tel.: 01221709989, E-mail: drahmedsaad@live.com, ahmed.saad@fmed.bu.edu.eg

ISSN: 2090-7265, February 2021, Vol.11, No. 1

\section{INTRODUCTION}

The slow-freezing method was used in humans after success of cryopreservation of mouse embryos in $1972^{[1]}$. The first successful frozen thawed human embryo pregnancy was reported in $1983^{[2]}$. For each patient, only a small number of embryos can be cryopreserved. Then a more easier and rapid technique for cryopreservation was needed. This alternative new method for human cryopreservation and vitrification, was reported by Rall and Fahy ${ }^{[3]}$. A small amount of medium harboring a high concentration of cryprotectant was used in this method to induce a glass-like state for rapid embryo cryopreservation then submersion into liquid nitrogen, thus preventing ice crystals formation ${ }^{[4]}$.

The many documented papers on the success of vitrification along with less procedure time than the slow freezing and with the developing experience on the procedure made it the procedure of choice by many IVF centers in storing both oocytes and embryos ${ }^{[5-8]}$.

As time goes by, frozen embryo transfer procedures have increased with more and more pregnancy rate as good as conventional fresh embryo transfer ${ }^{[9,10]}$ with publication showing that the frozen embryo transfer cases have lower preterm labour, birth weight and incidence of perinatal death than fresh cycles ${ }^{[11,12]}$.

Several vitrification kits are available in the market, but some of them result in inconsistent survival rates following thawing and others require extensive training ${ }^{[13,14]}$. Different combined cryoprotectants media, as ethylene glycol (EG), dimethyl sulfoxide (DMSO) and propanediol (PrOH), or EG alone have been reported for vitrification of human embryos and oocytes ${ }^{[15,16]}$.

The most frequently used cryoprotectant: Dimethyl sulphoxide (DMSO), is due to its rapid transport through 
cell membrane and it is highly efficient for oocyte, embryo and blastocyst vitrification. However, the possible toxicity of DMSO is of concern ${ }^{[17]}$.

There is also an evidence that a combined cryoprotectants might have better results than the media having a sole permeable cryoprotectant ${ }^{[18]}$. In fact, the first pregnancy and birth from Vitrified/thawed blastocyst transfer were achieved using EG and DMSO as cryoprotectants $^{[19,20]}$.

So, using a medium with EG, sucrose and DMSO as cryoprotectants for doing vitrification is both easy and competent for the cryopreservation of day 3 , day 4 morulae or day $5 / 6$ blastocysts ${ }^{[21,22]}$. Other formulations are DMSO-free, which are volume independent. Larger straws are much simple to use and allow faster embryo exposure to vitrification solutions (e.g. Global Fast Freeze kit, LifeGlobal, Canada $)^{[23]}$.

In this present study, we compared the two different vitrification and thawing media Home-made (HM) and Commercially-made (CM) in human blastocysts in intracytoplasmic sperm injection (ICSI) cycles.

We evaluated and compared survival, re-expansion, and percentage of live cells following vitrification and warming of Day 5 and Day 6 human blastocysts, vitrified and warmed with the Vit Kit Freeze/Thaw (Irvine Scientific, CA), or with two protocols using the Global Fast Freeze/Thaw Kits (LifeGlobal, Canada). Furthermore, cell survival of the vitrified/thawed embryos was compared with non-vitrified controls.

\section{PATIENTS AND METHODS}

Patients : This prospective study consisted of a total of 78 couples who had cryopreserved embryo transfer and vitrification of blastocysts after ICSI at a private fertility center, Banha city, Egypt between October 2015 and September 2018.

The mean age of all patients was 22-37 $(28.6 \pm 3.9)$. All patients included in this study were informed and gave a written consent for the cryopreservation procedure.

Experimental design : As shown in Table 1, a total of 154 blastocysts from 78 patients were randomly allocated into 2 treatment groups ; group 1 blastocysts were vitrified using the Global Fast Freeze Kit (Life Global; Commercial media) and thawed using Global Fast Freeze Thaw Kit (Life Global; Commercial media).

However, group 2 blastocysts were vitrified using Shady Grove Fertility RSC protocol in a Home-made media and thawed as was described by Kuwayama in HM in a Home-made media ${ }^{[24]}$.

After blastocysts were vitrified/thawed according to the protocols described below, they were assessed for immediate survival, then cultured for a period of 6-8 hrs and reassessed for survival and expansion then prepared for embryo transfer.

Embryo grading and digital imaging: Assessment of blastocyst morphological quality and stage (days 5 or 6) was done according to the classification developed by Gardner and Schoolcraft ${ }^{[25]}$.Digital images of each blastocyst were acquired before vitrification, immediately after thawing, and after 68 - $h$ culture, using a digital Watec camera connected to an inverted optical microscope with a thermal control microscope stage set on $37^{\circ} \mathrm{C}$.

Table 1: Distribution of embryos by stage in both treatment groups:

\begin{tabular}{|c|c|c|c|c|c|c|c|c|}
\hline \multirow{2}{*}{\multicolumn{2}{|c|}{ Treatment Groups }} & \multicolumn{7}{|c|}{ Blastocyst stage } \\
\hline & & 1 & 2 & 3 & 4 & 5 & 6 & Total \\
\hline 1 & $\begin{array}{l}\text { Commercial } \\
\text { media }\end{array}$ & 7 & 5 & 8 & 18 & 21 & 2 & 61 \\
\hline 2 & $\begin{array}{l}\text { Home-made } \\
\text { media }\end{array}$ & 4 & 9 & 13 & 25 & 37 & 5 & 93 \\
\hline
\end{tabular}

\section{Vitrification-warming procedures: Group 1 (CM)}

Vitrification procedure: Blastocysts were vitrified using the Global Fast Freeze Kit (LifeGlobal LLC, Guilford, CT, USA) which consists of 3 vitrification solutions, containing a combination of glycerol, ethylene glycol, human serum albumin (HSA), HEPES, and the base components of Global medium. Blastocysts were transferred to a drop of vitrification solution 1 , and left for $5 \mathrm{~min}$ and subsequently to vitrification solution 2 where they were left for another $5 \mathrm{~min}$, at room temperature. The blastocyst was then transferred and washed in several drops of vitrification solution 3 and immediately placed on the tip of the Cryotop (Kitazato, Japan) in a volume of $<1 \mu 1$ a few millimeters back from the black tip. The straws were then plunged directly into liquid nitrogen (LN) and then were restored in specific labeled places inside the LN tanks. This loading technique was done the same for both groups.

Warming procedure: Blastocysts were thawed using the Global Fast Freeze Thaw Kit (LifeGlobal LLC, Guilford, CT, USA) which consists of 3 solutions, with 
decreasing concentrations of sucrose. The protective cap of the straw was removed inside LN then transferred quickly and the blastocysts recovered into thawing solution 1 . After $2 \mathrm{~min}$, the blastocysts were transferred consecutively to thawing solution 2 ( 2 droplets) and were held for 2 minutes in each drop, then they were transferred to thawing solution 3 ( 3 droplets), where they were held for 3 min each. All procedures were done at room temperature, except for the last step where blastocysts were placed in the thawing solution 3, the dish was moved to a warm surface, at $37^{\circ} \mathrm{C}$. After thawing, all blastocysts were transferred to a pre-equilibrated dish containing $20 \mu \mathrm{l}$ culture drops of Global Total medium (LifeGlobal) under mineral oil and incubated $6-8$ at $37^{\circ} \mathrm{C}$ in standard incubation conditions ( $6 \% \mathrm{CO}_{2}$ in air) in order to assess post-thawing survival and expansion.

\section{Group 2 (HM)}

Vitrification procedure: was performed according to the method that was described by Shady Grove Fertility Reproductive Science Center ${ }^{[26]}$. In this protocol, 5 minutes prior to starting the vitrification process, expanded and hatching blastocysts were 'collapsed' artificially using one to two laser pulses directed at the trophectoderm at a site away from the inner cell mass.

\section{Vitrification solutions}

$$
\begin{aligned}
& \text { A-36ml mHTF }+9 \mathrm{ml} \text { SPS }(\mathrm{mHTF}+20 \% \mathrm{SPS})[\mathrm{WS}] \\
& \mathrm{B}-2.25 \mathrm{ml} \mathrm{EG}+2.25 \mathrm{ml} \mathrm{DMSO}+10.5 \mathrm{ml} \mathrm{A}(15 \% \mathrm{EG} / \\
& \mathrm{DMSO}+\mathrm{mHTF}) \\
& \text { C-7.5ml A }+7.5 \mathrm{ml} \mathrm{B}(7.5 \% \mathrm{EG} / \mathrm{DMSO}+\mathrm{mHTF})[\mathrm{ES}] \\
& \text { D-7.5ml B }+1.2825 \mathrm{~g} \mathrm{SUC}(15 \% \mathrm{EG} / \mathrm{DMSO}+0.5 \mathrm{M} \\
& \text { SUC) }[\mathrm{VS}]
\end{aligned}
$$

At room temperature blastocysts were put into drop 1(WS), then drop 1 (WS) connected with drop 2 (ES) for 2 minutes, then drop 2 (ES) was connected with drop 3 (ES) and waited 1min, drop 3 (ES) connected with drop 4 (ES) and waited $1 \mathrm{~min}$. Then Blastocysts were transferred to drop 5 (ES), and waited for re-expansion to $60-90 \%$ of the original volume. Then Blastocysts were moved into drops (VS) with minimal transfer of ES, and gently aspirated up and down to wash off the ES. Blastocysts were kept in VS for $45 \mathrm{secs}$. Then blastocysts were loaded and stored as described previously with group 1 .

Warming procedure: Thawing of blastocysts was done according to Kuwayama in HM [15] by placing the Cryotop in $1 \mathrm{ml}$ of Thawing solution 1 (3.44 g sucrose/10 ml Global total hepes media) for $50-60 \mathrm{~s}$ at $37{ }^{\circ} \mathrm{C}$ and moved into a $0.5 \mathrm{ml}$ of Thawing solution $2(1.72 \mathrm{~g}$ sucrose $/ 10 \mathrm{ml}$ Global total hepes media) for $3 \mathrm{~min}$, then to $0.5 \mathrm{ml}$ Thawing solution 3 ( $0.86 \mathrm{~g}$ sucrose $/ 10 \mathrm{ml}$ Global total hepes media) for 5 minutes, then to $0.5 \mathrm{ml}$ Thawing solution $4(0.43 \mathrm{~g}$ sucrose $/ 10 \mathrm{ml}$ Global total hepes media) for 5 minutes.
All procedures were done at room temperature, except the last step where blastocysts were placed in the thawing solution 4 , the dish was moved to a warm surface, at $37^{\circ} \mathrm{C}$.

After thawing, all blastocysts were transferred to a pre-equilibrated dish containing $20 \mu \mathrm{l}$ culture drops of Global Total medium (LifeGlobal) under mineral oil and incubated $6-8$ at $37^{\circ} \mathrm{C}$ in standard incubation conditions ( $6 \% \mathrm{CO} 2$ in air) in order to assess post-thawing survival and expansion same like group 1.

\section{Preparation of Patients' endometrium for embryo transfer:}

Patient's endometrium was prepared initially by the administration of the white tab. (2mg estradiol valerate) (Cyclo-Progynova, Bayer Schering pharma, Germany) ( $2 \mathrm{mg}$ three times- a day, initiated on the $2^{\text {nd }}$ day of the menstrual cycle), then endometrial assessment with vaginal ultrasound is done. If the endometrial thickness reaches $\geq 8 \mathrm{~mm}$ and is triple in shape, we will start the progesterone if not then follow up every 2 days till the endometrial thickness is $\geq 8 \mathrm{~mm}$.

Then, we give progesterone in the form of Pronotogest ampoule( progesterone $100 \mathrm{mg} / 2 \mathrm{ml}$, Produced by Nile Company for Pharmaceuticals, for: Marcyrl Co.) daily for 5 days then we do embryo transfer using soft transfer catheter (Labotect, Germany) under ultrasound guidance and continue with the treatment for 15 days and then a quantitative pregnancy test is done. If positive pregnancy test, assessment by ultrasound will be done after 10 days and the treatment ( Cycloprogynova and Prontogest) will be continued till at least the $7^{\text {th }}$ week and if needed till the $14^{\text {th }}$ week.

\section{STATISTICAL ANALYSIS}

Data are shown as mean values $\pm \mathrm{SD}$, The effect of Vitrification protocol on the blastocyst immediate survival, survival and expansion at 6-8 hrs after thawing and other post transfer evaluations were evaluated using Pearson's Chi-square test.

\section{RESULTS}

The representative digital images of blastocysts before vitrification, immediately after thawing and 6-8 hrs later in both study groups [CM] and [HM] are shown in Fig. 1.

The differences between groups regarding age and years of infertility the mean age of the patients was 22-36 (28.42 \pm 3.79$)$ for commercial media and 22-37 (28.71 \pm 4.16) for home-made media, respectively. The mean value of Duration of Infertility (years) Range for patients of CM was $1-6(2.73 \pm 1.15)$ and 1-5 (2.7 \pm 0.94$)$ for HM patient group (Table 2). 
There were no significant differences between two vitrification-thawing protocols (Group 1: CM, Group 2: $\mathrm{HM}$ ) on immediate survival. Using CM, vitrified/warmed Blastocysts had a survival rate of $98.3 \%(\mathrm{n}=60 / 61)$, while for HM, it was $98.9 \%$ ( $\mathrm{n}=92 / 93 ; P=0.27$ ), survival rate at $6-8 \mathrm{hrs}$ with CM $96.7 \% \quad(n=59 / 61)$, while for HM, it was $97.8 \% \quad(\mathrm{n}=91 / 93 ; \quad P=0.48)$, or expansion for $\mathrm{CM}$ $93.4 \% \quad(\mathrm{n}=57 / 61)$, while for $\mathrm{HM}$, it was $95.7 \%$ $(\mathrm{n}=92 / 93 ; P=0.39)$ after $6-8 \mathrm{~h}$ of culture following thawing (Fig. 2).

A total of 154 blastocysts from 78 patients were vitrified during this study and these vitrified embryos were thawed to perform 79 transfer cycles. One patient had two transfer cycles because she didn't get pregnant in her first ET cycle. A positive serum hCG concentration was measured 14 days after embryo transfer in all patients. The chemical pregnancy rate for commercial kit was $73.3 \%(n=22 / 30)$ Vs $75 \%(n=6 / 48 ; P=0.74)$ for home-made media. The clinical pregnancy rate for $\mathrm{CM}$ was $70 \%(\mathrm{n}=21 / 30)$ Vs $70.8 \%(\mathrm{n}=34 / 48 ; P=0.84)$ for HM. Implantation rate for CM was $60 \%(\mathrm{n}=18 / 30)$ Vs $60.4 \%(n=29 / 48 ; P=0.89)$ was for HM. Of these 47 implantation cases, 45 went home with healthy babies (Table 3).

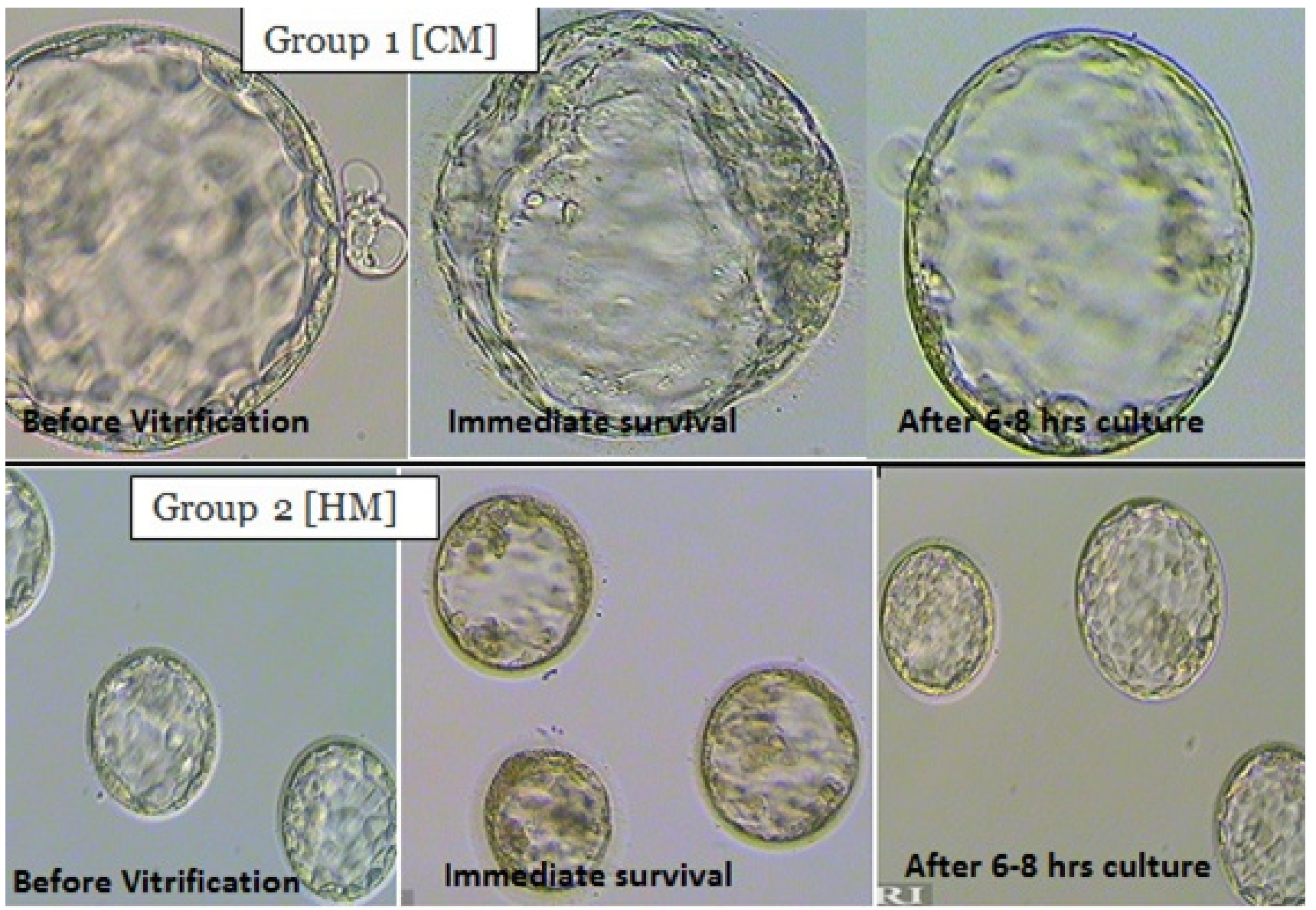

Fig. 1: Digital images of Blastocysts before vitrification and after thawing in both study groups. 


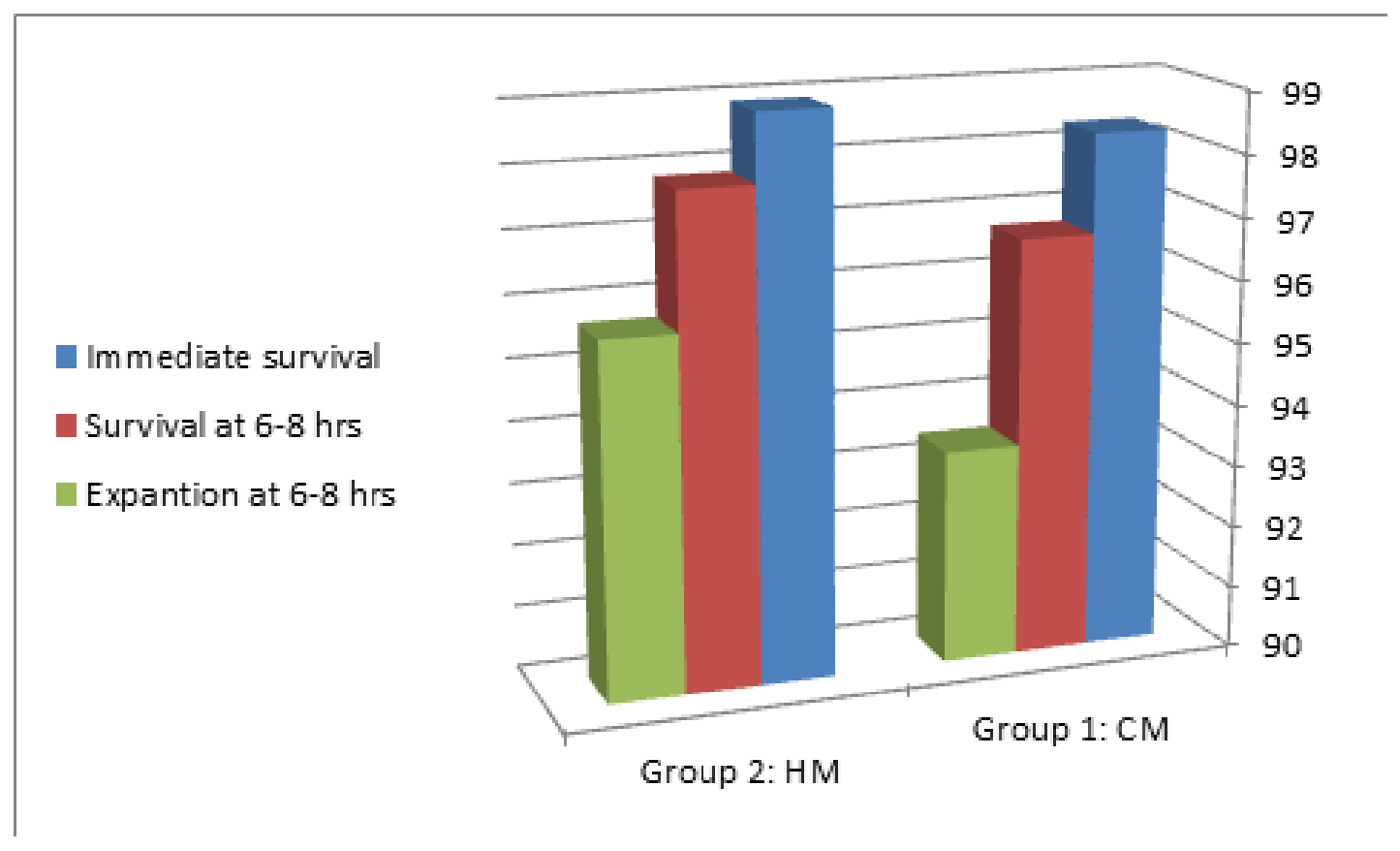

Fig. 2: Blastocyst survival and expansion following vitrification and thawing

Table 2: Difference between Groups regarding Initial Characteristics

\begin{tabular}{lccc}
\hline & Group 1 [CM] $(\mathrm{n}=30)$ & Group 2 [HM] $(\mathrm{n}=48)$ & $P^{*}$ \\
\hline Age (years) & $22-36$ & $22-37$ & 0.731 \\
Mean \pm SD & $28.42 \pm 3.79$ & $28.71 \pm 4.16$ & NS \\
Age (years) & $1-6$ & $1-5$ & 0.881 \\
Mean \pm SD & $2.73 \pm 1.15$ & $2.7 \pm 0.94$ & NS \\
\hline
\end{tabular}


Table 3: Main outcomes of the cycles of vitrified-thawed embryo transfer for both media

\begin{tabular}{|c|c|c|}
\hline Parameter & $\mathrm{CM}(\mathrm{n}=30)$ & $\mathrm{HM}(\mathrm{n}=48)$ \\
\hline No. of patients & 30 & 48 \\
\hline No. of cycles of embryo transfer & 30 & 49 \\
\hline No. of vitrified/warmed blastocysts & 61 & 93 \\
\hline No. of Blastocysts survived & 60 & 92 \\
\hline Survival rate (\%) & 98.3 & 98.92 \\
\hline No. of Blastocysts survived post $6-8 \mathrm{hrs}$ & 59 & 92 \\
\hline Survival rate post 6-8 hrs (\%) & 96.72 & 97.84 \\
\hline No. of Blastocysts expanded after 6-8 hrs & 57 & 89 \\
\hline Expansion rate $(\%)$ & 93.44 & 95.69 \\
\hline No. of chemical pregnancy (\%) & $22(73.3 \%)$ & $36(75 \%)$ \\
\hline No. of clinical pregnancies (\%) & $21(70 \%)$ & $34(70.83 \%)$ \\
\hline Implantation rate (\%) & $18(60 \%)$ & $29(60.42 \%)$ \\
\hline Incidence of twins & 2 & 3 \\
\hline Incidence of triplet & 1 & 3 \\
\hline No. of spontaneous abortions & 3 & 4 \\
\hline Live birth (\%) & $15(83.33 \%)$ & $25(86.20 \%)$ \\
\hline
\end{tabular}

CM: Commercial Media

HM: Home made Media 


\section{DISCUSSION}

The results found that vitrification did not affect blastocyst survival in any of the treatment groups. These findings confirm earlier results, which showed that thawed blastocysts which survived vitrification weren't different from fresh blastocysts, in terms of quality, DNA and chromosome integrity, ultrastructure, and developmental competence ${ }^{[27-29]}$.

Immediate survival, survival and re-expansion after 6-8 hrs, percentages of implantation and live birth rates were not significantly different for vitrified/thawed blastocysts between the two groups of HM and CM. This was observed despite the fact that the embryos were vitrified in different distinct developmental stages in the two groups, which may have negative effect on the development and impaired embryo health.

Neither survival, expansion nor percentage of implantation was significantly different between the two groups of the study. The results obtained with the Fast Freeze kit were in accordance with those observed in a preliminary study where a small sample of human blastocysts donated for research were vitrified with basis of the Global Fast Freeze media, and subsequently stained, leading to a survival rate following warming of $84 \%$ and a cell survival rate of $87 \%{ }^{[30]}$. Furthermore, the results of re-expansion rates, which is a positive prognostic marker associated with significantly increased implantation and clinical pregnancies, ${ }^{[31]}$ were similar.

It is well-established that Vitrification solutions that contain DMSO did not lead to cell membrane damage and death as quickly as the DMSO free vitrification solutions. However, those negative effects became apparent only after $10 \mathrm{~min}$ or longer exposure to the vitrification solution ${ }^{[32]}$. With global fast freeze kit protocol the embryos are exposed to Vit. solution 1 for 5 minutes and to Vit. solution 2 for 5 minutes, maximum 10 minutes as documented.

Concerning to group 2 [HM]; the results confirmed that ethylene glycol combined with DMSO, with its low toxicity and high permeability ${ }^{[33]}$, diffuses into the embryos and leaves them very rapidly owing to its low molecular weight. Thus embryos may undergo less osmotic stress during vitrification and thawing. Glycerol has a higher molecular weight and moves across the plasma membrane through aquaporins 3 that predominantly facilitates diffusion ${ }^{[34]}$. As earlier demonstrated by Stachecki and Cohen ${ }^{[14]}$, those 3 mixed materials are efficient cryoprotectants for blastocyst Vitrification.
The results also confirmed the previous findings that indicated that the homemade vitrification solution gave good results ${ }^{[35]}$.

\section{CONCLUSION}

The two vitrification protocols, commercial kit and home-made solution, did not differ in blastocysts survival, re-expansion and implantation rates. So, both methods could be used with equal success using simplified protocols and freezing straws which are cheaper and easy to load. HM it is providing an equal safety, and efficiency with lower cost in compared to CM.

\section{CONFLICT OF INTEREST}

There are no conflicts of interests.

\section{REFERENCES}

1. Whittingham DG, Leibo SP, Mazur P. Survival of mouse embryos frozen to -196 degrees and -269 degrees C. Science.1972;178:411-414.

2. Trounson A, Mohr L. Human pregnancy following cryopreservation, thawing and transfer of an eightcell embryo. Nature. 1983;305:707-709.

3. Rall WF, Fahy GM. Ice-free cryopreservation of mouse embryos at -196 degrees $C$ by vitrification. Nature. 1985;313: 573-575.

4. Kuc P, Kuczynska A, Stankiewicz B, Sieczynski P, Matysiak J, Kuczynski W. Vitrification vs. slow cooling protocol using embryos cryopreserved in the 5th or 6th day after oocyte retrieval and IVF outcomes. Folia Histochem Cytobiol. 2010:48(1): $84(84-88)$

5. Loutradi KE, Kolibianakis EM, Venetis CA, Papanikolaou EG, Pados G, Bontis I, et al. Cryopreservation of human embryos by vitrification or slow freezing: a systematic review and meta-analysis. Fertil Steril. 2008;90:186-93.

6. Kolibianakis EM, Venetis CA, Tarlatzis BC. Cryopreservation of human embryos by vitrification or slowfreezing: which one is better? Curr Opin Obstet Gynecol. 2009;21:270-4. 
7. AbdelHafez FF, Desai N, Abou-Setta AM, Falcone T, Goldfarb J. Slow freezing, vitrification and ultra-rapid freezing of human embryos: a systematic review and meta-analysis. Reprod Biomed Online. 2010;20:209-22.

8. Lopes A, Frederickx V, Van kerkhoven G, Campo R, Puttemans P, Gordts S. Survival, re-expansion and cell survival of human blastocysts following vitrification and warming using two vitrification systems. J Assist Reprod Genet. 2015; 32: 83-90

9. Loutradi KE, Kolibianakis EM, Venetis CA, et al. Cryopreservation of human embryos by vitrification or slow freezing: a systematic review and meta-analysis. Fertil Steril. 2008;90:186-93.

10. Cobo A, de los Santos MJ, Castellò D, et al. Outcomes of vitrified early cleavage-stage and blastocyst-stage embryos in a cryopreservation program: evaluation of 3,150 warming cycles. Fertil Steril 2012;98:1138-46.

11. Pelkonen S., Koivunen R.M., Martikainen H.,Gissler M., Hartikainen L., Tiitinen A. Obstetric and perinatal outcome of children born after the transfer of cryopreserved and fresh embryos. FertilSteril. 2008; 90 (Suppl): 64-65.

12. Baker H., Garrett C., Breheny S., Healy D., Jaques A., Halliday J. Outcomes after art mainly occur with fresh not frozen embryos transfers: significance and implications. FertilSteril. 2008; 90 (Suppl):29.

13. Antinori M, Licata E, Dani G, Cerusico F, Versaci C, Antinori S. Cryotopvitrification of human oocytes results in high survival rate and healthy deliveries. Reprod Biomed Online. 2007; 14:72-79.

14. Matsunari H, Maehara M, Nakano K, Ikezawa Y, Hagiwara Y, Sasayama N, et al. Hollow fiber vitrification: a novel method for vitrifying multiple embryos in a single device. J Reprod Dev. 2012; 58:599-608.

15. Takahashi K., Mukaida T., Goto T., et al. Perinatal outcome of blastocyst transfer with vitrification using cryoloop: a 4-year follow-up study. FertilSteril. 2005; 84: 88-92.

16. Chian R.C., Kuwayama M., Tan L., et al. High survival rate of bovine oocytes matured in vitro following Vitrification. J ReprodDev. 2004; 50 : 685-696.

17. Ali J, Shelton JN. Design of vitrification solutions for the cryopreservation of embryos. J ReprodFertil. 1993; 99:471-7.

18. Vajta G., Lewis I.M., Kuwayama M., et al. Sterile application of open pulled straw (OPS) vitrification method. Cryo-Letters. 1998; 19: 389-392.

19. Yokota Y, Sato S, Yokota M, Ishikawa Y, Makita M, Asada T, et al. Successful pregnancy following blastocyst vitrification: case report. Hum Reprod. 2000; 15:1802-1803.

20. Yokota Y, Sato S, Yokota M, Yokota H, Araki Y. Birth of a healthy baby following vitrification of human blastocysts. FertilSteril. 2001; 75:1027-1029.

21. Mukaida T., Nakamura S., Tomiyama T., Wada S., Oka C., Kasai M., et al. Vitrification of human blastocysts using cryoloops: clinical outcome of 223 cycles. Hum Reprod. 2003;18:384-391.

22. Desai N., Blackmon H., Szeptycki J., et al. Cryoloopvitrification of human day 3 cleavagestage embryos: post-vitrification development; pregnancy outcomes and live birth. Reprod Biomed Online. 2007; 14: 208-213.

23. Stachecki JJ, Cohen J. S3 vitrification system: a novel approach to blastocyst freezing. J ClinEmbryol. 2008; 11:5-14.

24. M. Kuwayama, G. Vajta, S. Ieda, O. Kato. Comparison of open and closed methods for vitrification of human embryos and the elimination of potential contamination. Reprod Biomed Online. 2005; 11:608-614.

25. Gardner DK, Schoolcraft WB. In vitro culture of human blastocyst. In: Jansen R, Mortimer D, editors. Towards reproductive certainty: infertility and genetics beyond. Carnforth: Parthenon Press. 1999; 378-88.

26. Lim JH, VerMilyea MD, Graham JR, Levy, MJ, Tucker MJ. Oocyte embryo cryopreservation. J Assist Reprod Genet. 2011; 28(11):997-1005.

27. Desai NN, Goldberg JM, Austin C, Falcone T. The new Rapid-i carrier is an effective system for 
human embryo vitrification at both the blastocyst and cleavage stage. ReprodBiolEndocrinol. 2013; 11:41.

28. AbdelHafez F, Xu J, Goldberg J, Desai N. Vitrification in open and closed carriers at different cell stages: assessment of embryo survival, development. DNA integrity and stability during vapor phase storage for transport. BMC Biotechnol. 2011;11:29.

29. Ku PY, Lee RK, Lin SY, Lin MH, Hwu YM. Comparison of the clinical outcomes between fresh blastocyst and vitrified-thawed blastocyst transfer. J Assist Reprod Genet. 2012; 29:13531356.

30. Stachecki JJ, Garrisi J, Sabino S, Caetano JP, Wiemer KE, Cohen J. A new safe, simple and successful vitrification method for bovine and human blastocysts. Reprod Biomed Online. 2008; $17: 360-7$.

31. Ebner T, Vanderzwalmen $\mathrm{P}$, Shebl O, Urdl $\mathrm{W}$, Moser M, Zech NH, et al. Morphology of vitrified/warmed day-5 embryos predicts rates of implantation, pregnancy and live birth. Reprod Biomed Online. 2009;19:72-8.

32. Kartberg A., Hambiliki F., Arvidsson T., StavreusEvers A., Svalander P. Vitrification with DMSO protects embryo membrane integrity better than solution without DMSO. Reprod Biomed Online, 2008; 17: 378-384.

33. Yoon T.K., Kim T.J.,Park S.E., et al. Live birth after vitrification of oocytes in a stimulated in vitro fertilization-embryo-transfer program. FertilSteril. 2003; 79:1323-1326.

34. Yamaji Y., Valdez Jr.,Seki S., et al. Cryoprotectant permeability of aquaporin-3 expressed in Xenopus oocytes. Cryobiology. 2006; 53: 258-267.

35. Al-Hasani S., Ozmen B., Koutlaki N., Schoepper B., Diedrich K., Schultze-Mosgau A. 2007; Three years of routine vitrification of human zygotes: is it still fair to advocate slow-rate freezing? Reprod Biomed Online. 2007; 14:288-293. 